\title{
Compression of the Median Nerve by a Lipoma in the Distal Forearm Associated with Bilateral Carpal Tunnel Syndromes
}

\author{
Seu-ryang Jang ${ }^{1}$, Jin-gyu Choi ${ }^{1}$, Byung-chul Son ${ }^{1,2}$ \\ ${ }^{I}$ Department of Neurosurgery, Seoul St. Mary's Hospital, College of Medicine, The Catholic University of Korea, Seoul, \\ ${ }^{2}$ The Catholic Neuroscience Institute, College of Medicine, The Catholic University of Korea, Seoul, Korea
}

Although lipomas are benign fatty tumors that are common in humans, lipomas causing compressive neuropathy are rare. Furthermore, compressive neuropathy of the median nerve in the distal forearm just proximal to the carpal tunnel has never been reported. We report a very rare case of symptomatic lipoma causing median neuropathy in the distal forearm, associated with bilateral carpal tunnel syndromes.

Key Words: Carpal tunnel syndrome $\cdot$ Lipoma $\cdot$ Median nerve $\cdot$ Median neuropathy

\section{INTRODUCTION}

Lipomas are the most common benign tumors in limbs ${ }^{4,19)}$. Although lipomas can occur in the vicinity of the peripheral nerve and occurrence of lipoma causing carpal tunnel syndrome has been sporadically reported, symptomatic compression of the median nerve by lipoma in the distal forearm is extremely rare. The authors report a rare case of lipoma compressing the median nerve in the distal forearm associated with bilateral carpal tunnel syndromes.

\section{CASE REPORT}

A 56-year-old, right-handed female patient presented with a 3-year history of progressively worsening paresthesia, numbness, and tingling in the lateral three digits and radial palm of both hands, with the right hand more severely affected. She described complete right-hand numbness after peeling potatoes, which was to some extent relieved by shaking the hand. She also described nocturnal numbness and frequent awakening during early morning for numbness. There was no neck pain or radicular symptom in her arms. Her medical history was unremarkable. Physical examination showed bilateral thenar muscle atrophy and muscle weakness of the abductor pollicis brevis, which was more prominent in the left hand.

Corresponding author: Byung-chul Son

Department of Neurosurgery, Seoul St. Mary's Hospital, The Catholic Neuroscience Institute, College of Medicine, The Catholic University of Korea, 222 Banpo-daero, Seocho-gu, Seoul 06591, Korea

Tel: +82-2-2258-6122, Fax: +82-2-594-4248

E-mail: sbc@catholic.ac.kr
Tendon reflexes were normal and symmetric. Decreased sensation to light touch and pinprick was evident in the lateral three digits of both hands. Tinel's sign was positive at both wrists, and a positive Phalen's test was present on the left. In addition to the typical pictures of median neuropathy, there was a solitary palpable mass with a doughy feel in the left distal forearm (Fig. 1A).

The electrodiagnostic findings were compatible with bilateral median entrapment neuropathy around the wrist, clinically carpal tunnel syndrome, severe degree on both sides by American Association of Electrodiagnostic Medicine (AAEM) classification ${ }^{1)}$. A magnetic resonance imaging (MRI) disclosed a round, well-marginated, homogeneous, high intensity mass on both T1- and T2 weighted images (Fig. 1B). The mass was about $3 \mathrm{~cm}$-in size and displacing the median nerve in the distal forearm just proximal to the left carpal tunnel. Considering the medical intractability and severe degree of the electrodiagnostic findings, bilateral carpal tunnel release and tumor removal were attempted.

After incision of the skin and superficial fascia of the distal forearm, the underlying lipoma, which was densely adherent to the bulged median nerve, and the median nerve was identified proximally and distally to the lipoma (Fig. 1C). After finding a cleavage plane between the lipoma and the median nerve under the microscopic vision, the lipoma was sharply dissected and resected under the microscopic vision (Fig. 1D). Because the lipoma was located just proximal to the classical entrapment site (carpal tunnel), the flexor retinaculum of the left carpal tunnel in the left hand was also decompressed. An open carpal tunnel release was also performed in the right hand because the patient had bilateral carpal tunnel syndromes and markedly hypertrophied flexor retinaculum was found and cut 

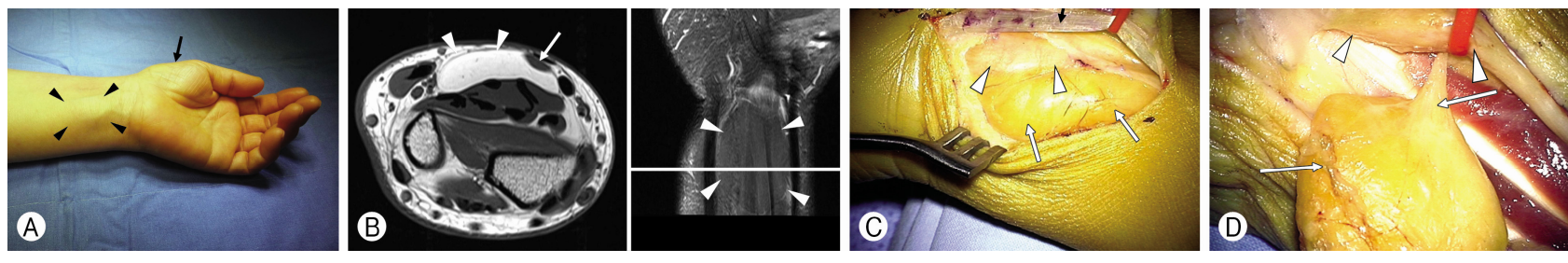

Fig. 1. (A) A photograph showing the location of the soft tissue mass (arrowheads) in the left distal forearm and the atrophy of the left thenar muscle (arrow). (B) An axial, T1-weighted magnetic resonance imaging (MRI) of the left forearm (left) showing the well-marginated, high signal intensity mass (arrowheads) and the median nerve (arrow) which was compressed and displaced to the radial side by the mass. A coronal, T2-weighted fat-suppression image (right) showing the location of the mass (arrowheads) in the distal forearm just proximal to carpal tunnel. (C) An intraoperative photograph showing the initial exposure of the lipoma (white arrows) which is adherent to the left median nerve (white arrowheads). The palmaris longus tendon (black arrow) is dissected and elevated. (D) An intraoperative photograph showing the final stage of the marginal dissection of the lipoma (white arrow) from the median nerve (white arrowheads). The compressed median nerve shows an hourglass deformity.

in the right side. Microscopic examination proved the mass to be mature fat with no evidence of structural and cellular atypism and there was a layer of synovial lining around it. The histological diagnosis was lipoma. Weakness of the left thenar muscles and numbness of the right hand improved progressively. However, the numbness of the finger-tips in the left hand was relieved by $-50 \%$ at 12 months after surgical intervention.

\section{DISCUSSION}

Soft-tissue tumors involving the peripheral nerves account for $1.02 \%$ to $4.9 \%$ of all tumors affecting the arm and hand ${ }^{26}$. There are two broad categories of soft-tissue tumors involving the nerves: neural sheath origin and nonneural sheath origin tumors $^{8,15)}$. Tumors of neural sheath origin are more frequent and account for more than half of the cases, while nonneural sheath origin tumors include several rare benign and malignant lesions, such as ganglions, hemangiomas, desmoids, ganglioneuromas, lymphangiomas, myoblastomas and lipomas ${ }^{15}$. Among the nonneural sheath-origin tumors, lipomas are a very rare cause of peripheral nerve compression ${ }^{8)}$. Therefore, peripheral nerve compression by lipoma is infrequently reported in the medical literature, and most reports are restricted to case reports and small series of patients $3,5,6,9-11,13,14,17,18,20,21,23-25,27$.

Lipomas are benign fatty tumors that are common in humans; it is a globose or ovoid mass which is mostly localized in the subcutaneous tissue ${ }^{15)}$. They are subclassified according to the anatomic site of the fat cells into dermal, subcutaneous, and subfascial ${ }^{8,15)}$. The subfascial type is also denominate parosteal lipoma. In general, there are 4 conditions wherein these tumors can affect a peripheral nerve: (1) solitary lipomas can eventually compress a nerve; (2) encapsulated lipomas can be located in the nerve (an intrinsic lesion); (3) lipofibromatous hamartoma, in which there is a fatty fibrous mass within the nerve; and (4) macrodystrophia lipomatosa, which produce an overgrowth of the extremities, especially the hand and fingers ${ }^{15)}$.

The most frequent clinical presentation of lipoma is a solitary lipoma. Two situations has been suggested for compression of a peripheral nerve by lipomas: (1) the nerve can be compressed by a subcutaneous lipoma on the point which it runs superficially, or (2) the lesion can be derived from deeper-seated fatty tissues (subfascial, parosteal lipomas), compressing nerves in more profound locations ${ }^{13)}$. The deep-seated, subfascial lipomas has been reported to have a preponderance of their association with radial nerve compression ${ }^{8,25}$. The frequency of the affected nerve is variable ${ }^{8}$. The most reported nerve affected by lipoma is the radial nerve, which is usually affected with subfascial, parosteal lipomas ${ }^{7,12,16,25}$, followed by the median ${ }^{5,6,8,10,14,18,22,27)}$ and ulnar nerves ${ }^{9,21)}$. Involvement of the nerves in the upper extremity is more common than those in the lower extremity and the reports regarding involvement of the median nerve by a lipoma within carpal tunnel are now increasing ${ }^{5,6,14,18,22)}$. Although symptomatic involvement of the median nerve in the proximal forearm by a lipoma has been reported twice ${ }^{13,27}$, to our knowledge, the case presented is the first of compression of the median nerve by a lipoma in the distal forearm just proximal to the wrist.

As shown in the present case, identification of a mass during the physical examination is important as tumors outside the typical points of nerve entrapment ${ }^{8)}$. Positive Tinel's sign over the lesion was suggested to facilitate identification of the tumor as the source of the nerve compression ${ }^{8)}$. Ultrasound is a simple and useful imaging method in the diagnosis of these tumors, especially in those cases wherein the lesion was superficially located ${ }^{8}$. However, MRI could provide more information in those situations in which greater number of structures may have been affected by the lesions, such as brachial plexus or popliteal fossa involvement ${ }^{8}$.

Not all lipomas of the wrist and hand are preoperatively diagnosed, and their diagnostic rate in the hand region is not known ${ }^{18)}$. The risk of neurologic deficit and iatrogenic sever- 
ing of the flexor pollicis longus tendon has been reported in a patient with giant lipoma of the hand who was not adequately investigated and treated with two carpal tunnel releases ${ }^{18)}$. In the present case, bilateral idiopathic carpal tunnel syndromes were found to be associated with median nerve compression in the left distal forearm just proximal to the carpal tunnel, according to physical examinations, an electrodiagnostic study, and a preoperative MRI scan.

Marginal resection with preservation of the neurovascular structure is the procedure of choice for lipomas, and more aggressive surgery is required in cases of malignant tumors ${ }^{8,15,19)}$. The surgical treatment of lipomas demonstrated good outcomes in pain relief and neurological recovery in most of the cases ${ }^{8,15,19)}$. The importance of proper exposure of the involved nerve proximal and distal to the lesion to ensure safe resection has been emphasized. The neural elements should be identified and protected proximally and distally to the tumor itself prior to attempting direct resection ${ }^{2}$.

\section{CONCLUSION}

Compressive neuropathy of the median nerve by a lipoma is very rare. We present a case of compression of the median nerve in the distal forearm associated with typical carpal tunnel syndrome. The need for a high index of suspicion in conjunction with detailed clinical examination and appropriate investigational studies is evident.

\section{REFERENCES}

1. American Association of Electrodiagnostic Medicine, American Academy of Neurology, American Academy of Physical Medicine and Rehabilitation: Practice parameter for electrodiagnostic studies in carpal tunnel syndrome: summary statement. Muscle Nerve 25:918-922, 2002

2. Barbour JR, Boyd KU. Tumors of the peripheral nervous system. In: Mackinnon SE, Yee A eds. Peripheral nerve surgery. New York, NY: Thieme, 2014, pp530-571

3. Bieber EJ, Moore JR, Weiland AJ: Lipomas compressing the radial nerve at the elbow. J Hand Surg Am 11:533-535, 1986

4. Capelastegui A, Astigarraga E, Fernandez-Canton G, Saralegui I, Larena JA, Merino A: Masses and pseudomasses of the hand and wrist: MR findings in 134 cases. Skeletal Radiol 28:498-507, 1999

5. Chen $\mathrm{CH}$, Wu T, Sun JS, Lin WH, Chen CY: Unusual causes of carpal tunnel syndrome: space occupying lesions. J Hand Surg Eur Vol 37:14-19, 2012

6. Fazilleau F, Williams T, Richou J, Sauleau V, Le Nen D: Median nerve compression in carpal tunnel caused by a giant lipoma. Case Rep Orthop 2014:654934, 2014

7. Fitzgerald A, Anderson W, Hooper G: Posterior interosseous nerve palsy due to parosteal lipoma. J Hand Surg Br 27:535537, 2002

8. Flores LP, Carneiro JZ: Peripheral nerve compression secondary to adjacent lipomas. Surg Neurol 67:258-262, 2007

9. Galeano M, Colonna M, Risitano G: Ulnar tunnel syndrome secondary to lipoma of the hypothenar region. Ann Plast Surg 46:83-84, 2001

10. Goldstein LJ, Helfend LK, Kordestani RK: Postoperative edema after vascular access causing nerve compression secondary to the presence of a perineuronal lipoma: case report. Neurosurgery 50:412-413, 2002

11. Hazrati Y, Miller S, Moore S, Hausman M, Flatow E: Suprascapular nerve entrapment secondary to a lipoma. Clin Orthop Relat Res:124-128, 2003

12. Henrique A: A high radial neuropathy by parosteal lipoma compression. J Shoulder Elbow Surg 11:386-388, 2002

13. Higgs PE, Young VL, Schuster R, Weeks PM: Giant lipomas of the hand and forearm. South Med J 86:887-890, 1993

14. Jalan D, Garg B, Marimuthu K, Kotwal P: Giant lipoma: an unusual cause of carpal tunnel syndrome. Pan Afr Med J 9:29, 2011

15. Kline DG, Hudson AR. Tumors involving nerve. In: Kline DG, Hudson AR eds. Nerve injuries: Operative results for major nerve injuries, entrapments and tumors. Philadelphia, PA: W. B. Saunders, 1995, pp525-574

16. Lidor C, Lotem M, Hallel T: Parosteal lipoma of the proximal radius: a report of five cases. J Hand Surg Am 17:1095-1097, 1992

17. Nicklas BJ, Schwein J: Juxtamalleolar lipoma with intermediate dorsal cutaneous nerve entrapment. J Am Podiatr Med Assoc 81:564-566, 1991

18. Pagonis T, Givissis P, Christodoulou A: Complications arising from a misdiagnosed giant lipoma of the hand and palm: a case report. J Med Case Rep 5:552, 2011

19. Phalen GS, Kendrick JI, Rodriguez JM: Lipomas of the upper extremity. A series of fifteen tumors in the hand and wrist and six tumors causing nerve compression. Am J Surg 121:298-306, 1971

20. Resende LA, Silva MD, Kimaid PA, Schiavão V, Zanini MA, Faleiros AT: Compression of the peripheral branches of the sciatic nerve by lipoma. Electromyogr Clin Neurophysiol 37: 251-255, 1997

21. Sakai K, Tsutsui T, Aoi M, Sonobe H, Murakami H: Ulnar neuropathy caused by a lipoma in Guyon's canal--case report. Neurol Med Chir (Tokyo) 40:335-338, 2000

22. Sbai MA, Benzarti S, Msek H, Boussen M, Khorbi A: Carpal tunnel syndrome caused by lipoma: a case report. Pan Afr Med J 22:51, 2015

23. Sergeant G, Gheysens O, Seynaeve P, Van Cauwelaert J, Ceuppens $\mathrm{H}$ : Neurovascular compression by a subpectoral lipoma. A case report of a rare cause of thoracic outlet syndrome. Acta Chir Belg 103:528-531, 2003

24. Sonoda H, Takasita M, Taira H, Higashi T, Tsumura H: Carpal tunnel syndrome and trigger wrist caused by a lipoma arising from flexor tenosynovium: a case report. J Hand Surg Am 27: 1056-1058, 2002

25. Stagno d'Alcontres F, Pardo A, Stracuzzi G: On parosteal lipomas of the limbs. Minerva Chir 51:607-616, 1996

26. Strickland JW, Steichen JB: Nerve tumors of the hand and forearm. J Hand Surg Am 2:285-291, 1977

27. Valbuena SE, O'Toole GA, Roulot E: Compression of the median nerve in the proximal forearm by a giant lipoma: A case report. J Brachial Plex Peripher Nerve Inj 3:17, 2008 